Research Article

\title{
Infection Prevalence and Antibiotic Resistance Levels in Ureaplasma urealyticum and Mycoplasma hominis in Gynecological Outpatients of a Tertiary Hospital in China from 2015 to 2018
}

\author{
Wei Zhang $\mathbb{D}^{1},{ }^{1}$ Lijuan Li, ${ }^{1}$ Xuelian Zhang, ${ }^{2}$ Hongshu Fang, ${ }^{1}$ Huajian Chen, \\ and Changxian Rong $\mathbb{D D}^{2}$ \\ ${ }^{1}$ Department of Clinical Laboratory, The Fourth People's Hospital of Chongqing, Chongqing 400014, China \\ ${ }^{2}$ Department of Gynecology, The Fourth People's Hospital of Chongqing, Chongqing 400014, China \\ Correspondence should be addressed to Changxian Rong; wh19771002@163.com
}

Received 14 September 2020; Revised 7 December 2020; Accepted 29 December 2020; Published 13 January 2021

Academic Editor: Louis Detolla

Copyright (c) 2021 Wei Zhang et al. This is an open access article distributed under the Creative Commons Attribution License, which permits unrestricted use, distribution, and reproduction in any medium, provided the original work is properly cited.

\begin{abstract}
The aim of this study was to estimate the Ureaplasma urealyticum and Mycoplasma hominis infection prevalence and antibiotic resistance levels in gynecological outpatients. Clinical characteristics and laboratory data of gynecological outpatients of the Fourth People's Hospital of Chongqing from 2015 to 2018 were retrospectively analyzed. Antibiotic resistance levels in U. urealyticum and M. hominis were defined by a commercial Mycoplasma kit for antibiotic susceptibility testing. Univariate analysis and multivariate logistic regression analysis were performed to evaluate risk factors associated with Mycoplasma isolation. Comparisons of yearly distributions and resistance rates were assessed by chi-square tests. Fifty-six percent of gynecological outpatients were positive for U. urealyticum, and $11.02 \%$ were positive for $M$. hominis. In the univariate analysis, women aged 30-39 years or with a history of pregnancy or gynecological diseases had an increased risk for Mycoplasma isolation, while women who were postmenopausal or had an education level of undergraduate degree or above had a decreased risk of Mycoplasma isolation. In the multivariate logistic regression model, an independent risk factor for Mycoplasma isolation was a history of gynecological diseases, while a bachelor's degree, master's degree, or above were protective factors against Mycoplasma isolation. There were distinctly gradual increases in the positivity rates of U. urealyticum and M. hominis from 2015 to 2018 and an overall increasing trend of resistance to ten antibiotics among U. urealyticum and M. hominis. The top three antibiotics associated with resistance were ofloxacin, sparfloxacin, and levofloxacin. Doxycycline, josamycin, and minocycline were preferred because they had the lowest levels of resistance. Increases in the prevalence of infection and antibiotic resistance in $U$. urealyticum and M. hominis were observed from 2015 to 2018, clearly confirming the necessity to monitor the standardized administration of antibiotics.
\end{abstract}

\section{Introduction}

Ureaplasma urealyticum and Mycoplasma hominis belong to the class Mollicutes, genus Mycoplasma, which are the smallest known free-living microorganisms [1]. Genital Mycoplasma mainly consisting of $U$. urealyticum and M. hominis is found among the cervical flora of approximately $70 \%$ of women of child-bearing age [2]. Asymptomatic infections by $U$. urealyticum and $M$. hominis have been proven to be associated with increased risks of urogenital tract inflammation [3], female infertility [4], and adverse pregnancy outcomes [5]. Unlike conventional bacteria, Mycoplasma lacks a cell wall and thus is resistant to antibiotics that interfere with cell wall synthesis, such as penicillins, cephalosporins, and vancomycin, but they are usually susceptible to antibiotics that inhibit protein synthesis or suppress topoisomerases, such as quinolones, tetracyclines, and macrolides $[6,7]$. Some species of 
Mycoplasma are innately or selectively resistant to an antibiotic, while others are not. Some species initially sensitive to an antibiotic can become resistant. The extensive abuse of quinolones has led to a gradual increase in resistance of $U$. urealyticum and the incidence of fluoroquinolone-resistant strains in many countries [8-10]. Furthermore, the high detection rate and poor therapeutic efficacy for $U$. urealyticum and M. hominis are great challenges for clinicians and cause heavy economic and mental burdens on patients [11]. Here, we discuss the evolution of antibiotic resistance in $U$. urealyticum and $M$. hominis in gynecological outpatients from 2015 to 2018.

\section{Methods}

This retrospective study was performed at the Fourth People's Hospital of Chongqing (Chongqing, China). Ethical approval was obtained by the Ethics Committee of the Fourth People's Hospital of Chongqing. Routine gynecological examinations were conducted in accordance with regulations and guidelines. The clinical characteristics of outpatients in the Department of Gynecology from January 1,2015 , to December 31, 2018, including age, marital status, education level, occupation, smoking status, drinking status, gravidity, parity, menopause status, and history of gynecological diseases, were collected from the Hospital Information System (HIS).

Endocervical specimens were examined by the Department of Clinical Laboratory. All specimens were processed within one hour after collection. The identification and antibiotic susceptibility of $U$. urealyticum and M. hominis were determined by a commercial Mycoplasma ICS kit (Lizhu Biotech Co., Ltd., Zhuhai, China) according to the manufacturer's guidelines and standard operating procedures. All inoculated strips were incubated at $37^{\circ} \mathrm{C}$ in a $\mathrm{CO}_{2}$ incubator and then observed for color changes. The results of $U$. urealyticum and M. hominis detection were interpreted after 48 hours of incubation. The strips provided information on susceptibility to ten antibiotics including tetracycline, ofloxacin, doxycycline, josamycin, sparfloxacin, roxithromycin, minocycline, levofloxacin, clarithromycin, and azithromycin, at two concentrations, with three interpretations: susceptible $(S)$, intermediate $(I)$, and resistant $(R)$. The breakpoints were as follows $(\mathrm{mg} / \mathrm{L})$ : tetracycline $S \leq 4, R \geq 8$; ofloxacin $S \leq 1, R \geq 4$; doxycycline $S \leq 4, R \geq 8$; josamycin $S \leq 2, R \geq 8$; sparfloxacin $S \leq 1, R \geq 4$; roxithromycin $S \leq 1, R \geq 4$; minocycline $S \leq 4, R \geq 8$; levofloxacin $S \leq 1, R \geq 4$; clarithromycin $S \leq 1, R \geq 4$; azithromycin $S \leq 1$, $R \geq 4$.

Statistical analysis was performed using SPSS software 22.0. Prevalence and antibiotic resistance rates were calculated as frequencies $(n)$ and percentages (\%). Associations between clinical characteristics and Mycoplasma isolation were measured by the chi-square test, as well as associations between year and Mycoplasma isolation and associations between year and antibiotic resistance. Multivariate logistic regression analysis was used to assess the risk factors associated with Mycoplasma isolation. A $P$ value $<0.05$ was considered to be statistically significant.

\section{Results}

3.1. Clinical Characteristics of Gynecological Outpatients. A total of 5670 gynecological outpatients from 2015 to 2018 were included in this study, and the overall prevalence of Mycoplasma isolation was $56.53 \%$. The univariate analysis results of clinical characteristic associations are shown in Table 1. Approximately, half of the gynecological outpatients with Mycoplasma isolation were in the 20-29 year age range; however, Mycoplasma isolation had the highest frequency in the 30-39 year age range. Gynecological outpatients who were postmenopausal or had an undergraduate degree or above were less likely to develop Mycoplasma infection than their counterparts. The risk for Mycoplasma isolation was significantly increased in patients with a history of pregnancy or gynecological diseases. No association was found between Mycoplasma isolation and marital status, occupation, smoking status, drinking status, or parity.

3.2. Risk Factors for Mycoplasma Isolation. The risk factors for Mycoplasma isolation based on the multivariate logistic regression analysis are shown in Table 2 . In the multivariate model, an independent risk factor for Mycoplasma isolation was a history of gynecological diseases, while education levels of bachelor's degree, master's degree, and above were protective factors against Mycoplasma isolation. In addition, there were no associations between Mycoplasma isolation and age, gravidity, or menopausal status.

3.3. Yearly Distributions of U. urealyticum and M. hominis from 2015 to 2018. The distributions of Mycoplasma isolation between 2015 and 2018 are shown in Table 3. Among 5670 gynecological outpatients, 3205 (56.53\%) were positive for U. urealyticum, and $625(11.02 \%)$ were positive for $M$. hominis. There was a distinctly gradual increase in the positivity rates for U. urealyticum and M. hominis from 2015 to 2018. Furthermore, the prevalence rates of both $U$. urealyticum and $M$. hominis had obvious differences ( $P=0.018$ and $P=0.003$, respectively) according to the yearly distributions.

3.4. Antibiotic Resistance of U. urealyticum and M. hominis from 2015 to 2018. Table 4 shows the progression of antibiotic resistance of Mycoplasma from 2015 to 2018. There was an overall increasing trend of resistance to ten antibiotics among $U$. urealyticum and $M$. hominis; the resistance rates to ofloxacin as well as sparfloxacin, roxithromycin, levofloxacin, clarithromycin, and azithromycin were significantly different among the four years. The top three antibiotics associated with resistance were ofloxacin, sparfloxacin, and levofloxacin, with total resistance rates of $61.84 \%, 51.54 \%$, and $40.50 \%$, respectively. However, the resistance rates to tetracyclines, including tetracycline, doxycycline, and minocycline were always below 3\%, while those to macrolides, including roxithromycin, clarithromycin, and azithromycin, were approximately $20 \%$, except for josamycin, which had a less than $2 \%$ resistance 
TABLE 1: Clinical characteristics of gynecological outpatients.

\begin{tabular}{|c|c|c|c|c|c|c|}
\hline \multirow{2}{*}{ Characteristics } & \multirow{2}{*}{ Total } & \multicolumn{2}{|c|}{ Mycoplasma } & \multirow{2}{*}{ OR } & \multirow{2}{*}{$95 \%$ CI } & \multirow{2}{*}{$P$} \\
\hline & & Positive $(n=3205)$ & Negative $(n=2465)$ & & & \\
\hline \multicolumn{7}{|l|}{ Age } \\
\hline$\leq 19$ & 57 & $26(0.8)$ & $31(1.3)$ & & Reference & \multirow{5}{*}{0.019} \\
\hline $20-29$ & 2831 & $1608(50.2)$ & $1223(49.5)$ & 1.568 & $0.926-2.654$ & \\
\hline $30-39$ & 1660 & $947(29.5)$ & $713(28.9)$ & 1.584 & $0.932-2.691$ & \\
\hline $40-49$ & 1032 & $587(18.3)$ & $445(18.1)$ & 1.573 & $0.921-2.687$ & \\
\hline$\geq 50$ & 90 & $37(1.2)$ & $53(2.2)$ & 0.832 & $0.426-1.626$ & \\
\hline \multicolumn{7}{|l|}{ Marital status } \\
\hline Married & 4652 & $2561(79.9)$ & $1991(80.8)$ & \multicolumn{2}{|r|}{ Reference } & \multirow{2}{*}{0.417} \\
\hline Unmarried & 1018 & $644(20.1)$ & $474(19.2)$ & 1.056 & 0.925-1.206 & \\
\hline \multicolumn{7}{|l|}{ Education } \\
\hline High school and below & 2374 & $1632(50.9)$ & $1142(46.3)$ & & Reference & \multirow{3}{*}{0.003} \\
\hline Bachelor & 2239 & $1216(38.0)$ & $1023(41.5)$ & 0.832 & $0.743-0.931$ & \\
\hline Master and above & 1057 & $357(11.1)$ & $300(12.2)$ & 0.833 & $0.702-0.988$ & \\
\hline \multicolumn{7}{|l|}{ Occupation } \\
\hline Yes & 4371 & $2468(77.0)$ & $1903(77.2)$ & \multicolumn{2}{|r|}{ Reference } & \multirow{2}{*}{0.862} \\
\hline No & 1299 & $737(23.0)$ & $562(22.8)$ & 1.011 & $0.892-1.146$ & \\
\hline \multicolumn{7}{|l|}{ Smoking } \\
\hline No & 86 & $3048(95.1)$ & $2336(94.8)$ & \multicolumn{2}{|r|}{ Reference } & \multirow{2}{*}{0.568} \\
\hline Yes & 5584 & $157(4.9)$ & $129(5.2)$ & 0.933 & $0.734-1.185$ & \\
\hline \multicolumn{7}{|l|}{ Drinking } \\
\hline No & 632 & $2631(82.1)$ & $2007(81.4)$ & \multicolumn{2}{|r|}{ Reference } & \multirow{2}{*}{0.517} \\
\hline Yes & 5038 & $574(17.9)$ & $458(18.6)$ & 0.956 & 0.835-1.095 & \\
\hline \multicolumn{7}{|l|}{ Gravidity } \\
\hline 0 & 1040 & $781(24.4)$ & $659(26.7)$ & \multicolumn{2}{|r|}{ Reference } & \multirow{2}{*}{0.043} \\
\hline$\geq 1$ & 4630 & $2424(75.6)$ & $1806(73.3)$ & 1.133 & $1.004-1.277$ & \\
\hline \multicolumn{7}{|l|}{ Parity } \\
\hline 0 & 2156 & $1195(37.3)$ & $961(39.0)$ & \multicolumn{2}{|r|}{ Reference } & \multirow{2}{*}{0.191} \\
\hline$\geq 1$ & 3514 & $2010(62.7)$ & $1504(61.0)$ & 1.075 & 0.965-1.197 & \\
\hline Menopause status & & & & & & \\
\hline No & 297 & $3055(95.3)$ & $2318(94.0)$ & & Reference & 0.032 \\
\hline Yes & 5373 & $150(4.7)$ & $147(6.0)$ & 0.774 & $0.613-0.978$ & 0.032 \\
\hline History of gynecological & & & & & & \\
\hline No & 2361 & $1813(56.5)$ & $1496(60.7)$ & & Reference & \\
\hline Yes & 3309 & $1392(43.5)$ & $969(39.3)$ & 1.185 & $1.065-1.319$ & 0.002 \\
\hline
\end{tabular}

rate. Therefore, doxycycline, josamycin, and minocycline are recommended because they had relatively low resistance rates.

\section{Discussion}

This study was performed in a tertiary hospital in Chongqing, China, to evaluate the infection prevalence and antibiotic resistance levels of Mycoplasma in gynecological outpatients, as well as the clinical characteristics and risk factors. Overall, in the present study of 5670 gynecological outpatients, we found that $56.53 \%$ of outpatients were positive for Mycoplasma. Education level and history of gynecological diseases were independently associated with Mycoplasma isolation, and a history of gynecological diseases was a risk factor for Mycoplasma isolation, while a bachelor's degree, master's degree, and above were protective factors against Mycoplasma isolation. Although approximately half of the gynecological outpatients with Mycoplasma isolation were 20-29 years old, Mycoplasma isolation occurred most frequently in the 30-39-year age group, consistent with one study that showed that the proportions of $U$. urealyticum and M. hominis detected in the 30-39-year age group were higher than those detected in the other age groups [12]. A prospective, cross-sectional study found that Mycoplasma infection had statistically significant correlations with more than 4 sexual partners, coinfection with other sexually transmitted pathogens, inconsistent contraceptive use, abortion, and history of gynecological diseases [13]. However, no associations between Mycoplasma infection and marital status, occupation, smoking status, drinking status, or parity were found in this study.

The detection rate significantly increased by year for U. urealyticum, from $53.80 \%$ in 2015 to $59.33 \%$ in 2018 , and for M. hominis, from $8.71 \%$ in 2015 to $12.42 \%$ in 2018 . Several studies with similar aims have been performed in China, although they were limited to various years and specific populations. In a tertiary hospital in Beijing, China, between 2009 and 2013, the prevalence rates of 
TABLE 2: Multivariate logistic regression analysis of risk factors for Mycoplasma isolation.

\begin{tabular}{|c|c|c|c|c|c|c|}
\hline & $\beta$ & SE & Wald & Sig. & OR & $95 \% \mathrm{CI}$ \\
\hline \multicolumn{7}{|l|}{ Age } \\
\hline$\leq 19$ & \multicolumn{6}{|c|}{ Reference } \\
\hline $20-29$ & 0.438 & 0.270 & 2.625 & 0.105 & 1.549 & $(0.912,2.631)$ \\
\hline $30-39$ & 0.407 & 0.273 & 2.219 & 0.136 & 1.502 & $(0.879,2.565)$ \\
\hline $40-49$ & 0.394 & 0.278 & 2.002 & 0.157 & 1.483 & $(0.859,2.559)$ \\
\hline$\geq 50$ & -0.193 & 0.376 & 0.264 & 0.608 & 0.825 & $(0.395,1.722)$ \\
\hline \multicolumn{7}{|l|}{ Education } \\
\hline High school and below & \multicolumn{6}{|c|}{ Reference } \\
\hline Bachelor & -0.196 & 0.058 & 11.596 & 0.001 & 0.822 & $(0.734,0.920)$ \\
\hline Master and above & -0.217 & 0.088 & 6.085 & 0.014 & 0.805 & $(0.678,0.956)$ \\
\hline \multicolumn{7}{|l|}{ Gravidity } \\
\hline 0 & \multicolumn{6}{|c|}{ Reference } \\
\hline$\geq 1$ & 0.102 & 0.064 & 2.549 & 0.110 & 1.107 & $(0.977,1.255)$ \\
\hline \multicolumn{7}{|l|}{ Menopause status } \\
\hline No & \multicolumn{6}{|c|}{ Reference } \\
\hline Yes & -0.157 & 0.154 & 1.037 & 0.309 & 0.854 & $(0.631,1.157)$ \\
\hline \multicolumn{7}{|c|}{ History of gynecological diseases } \\
\hline No & \multicolumn{6}{|c|}{ Reference } \\
\hline Yes & 0.182 & 0.057 & 10.230 & 0.001 & 1.200 & $(1.073,1.341)$ \\
\hline
\end{tabular}

TABLE 3: Yearly distributions of U. urealyticum and M. hominis from 2015 to 2018.

\begin{tabular}{lccc}
\hline Year & Total & U. urealyticum & M. hominis \\
\hline $2015-2018$ & 5670 & $3205(56.53)$ & $625(11.02)$ \\
2015 & 1251 & $673(53.80)$ & $109(8.71)$ \\
2016 & 1275 & $704(55.22)$ & $128(10.04)$ \\
2017 & 1509 & $858(56.86)$ & $185(12.26)$ \\
2018 & 1635 & $970(59.33)$ & $203(12.42)$ \\
$\chi^{2}$ & - & 20.16 & 27.67 \\
$P$ & - & 0.018 & 0.003 \\
\hline
\end{tabular}

TABle 4: Antibiotic resistance of U. urealyticum and M. hominis from 2015 to 2018.

\begin{tabular}{|c|c|c|c|c|c|c|c|}
\hline Antibiotic & 2015-2018 & 2015 & 2016 & 2017 & 2018 & $x^{2}$ & $P$ \\
\hline Tetracycline & $77(2.40)$ & $12(1.78)$ & 14 (1.99) & $24(2.80)$ & $27(2.78)$ & 2.786 & 0.426 \\
\hline Ofloxacin & $1982(61.84)$ & $247(36.70)$ & $388(55.11)$ & $593(69.11)$ & $754(77.73)$ & 316.8 & $<0.001$ \\
\hline Doxycycline & $51(1.59)$ & $12(1.78)$ & 7 (0.99) & $13(1.52)$ & $19(1.96)$ & 2.628 & 0.453 \\
\hline Josamycin & $32(1.00)$ & $6(0.89)$ & $4(0.57)$ & $10(1.17)$ & $12(1.24)$ & 2.198 & 0.532 \\
\hline Sparfloxacin & $1652(51.54)$ & $228(33.88)$ & $364(51.70)$ & $514(59.91)$ & $545(56.19)$ & 116.5 & $<0.001$ \\
\hline Roxithromycin & $510(15.91)$ & $57(8.47)$ & $84(11.93)$ & $172(20.05)$ & $197(20.31)$ & 61.17 & $<0.001$ \\
\hline Minocycline & $48(1.50)$ & $9(1.34)$ & $9(1.28)$ & $15(1.75)$ & $15(1.55)$ & 0.728 & 0.867 \\
\hline Levofloxacin & $1298(40.50)$ & $108(16.05)$ & $189(26.85)$ & $420(48.95)$ & $581(59.90)$ & 398.3 & $<0.001$ \\
\hline Clarithromycin & $401(12.51)$ & $48(7.13)$ & $69(9.80)$ & $144(16.78)$ & $140(14.43)$ & 40.09 & $<0.001$ \\
\hline Azithromycin & $454(14.17)$ & $49(7.28)$ & $75(10.65)$ & $160(18.65)$ & $169(17.42)$ & 56.12 & $<0.001$ \\
\hline
\end{tabular}

$U$. urealyticum and $M$. hominis in female outpatients were $33.1 \%$ and $2.6 \%$, respectively [12]. A prospective study in Baoding, China, from 2013 to 2014 found a U. urealyticum prevalence of $57.60 \%$ in female outpatients with urogenital infections [14]. A community-based cross-sectional study in Shanghai, China, from 2009 to 2014 showed that the $U$. urealyticum infection rate in vaginal secretions was $43.62 \%$ in women with urinary tract infections [15]. The wide-ranging prevalence of $U$. urealyticum and $M$. hominis in previous studies could be attributed to variations in study designs, study populations, sampling sites, and laboratory methods [16].

Quinolones are extensively used for the treatment of urogenital and gastrointestinal infections; they prevent pathogen DNA from unwinding and duplicating by inhibiting the type II topoisomerase DNA gyrase and topoisomerase IV [17]. A large number of studies have shown that the Mycoplasma resistance rate to quinolones is much higher than those to other antibacterials, and resistance is increasing worldwide [18-20]. For example, the 
U. urealyticum resistance rate to ofloxacin increased from $24.1 \%$ in 1999 to $66.8 \%$ in 2004 [18]. U. urealyticum and $M$. hominis had resistance rates of $50.6 \%$ and $17.7 \%$ to ofloxacin, respectively, between 2011 and 2015 [20]. Consistently, in this study, ofloxacin, sparfloxacin, and levofloxacin, which are all quinolones, were the top three antibiotics associated with resistance, accounting for approximately $50 \%$ of the total resistance; their rates were much higher than those associated with tetracyclines and macrolides. Notably, there was a gradually increasing prevalence of quinolone resistance from 2015 to 2018. The mechanism of quinolone resistance involves mutations in the gyrA and gyrB subunits of DNA gyrase and parC and parE subunits of topoisomerase IV that probably arise because of antimicrobial selective pressure $[17,21]$. Wang et al. reported that nemonoxacin, a novel nonfluorinated quinolone, may have increased antibacterial activity and decreased adverse effects on account of its C-8-methoxy structure on the quinolone ring [22]. The resistance rates for tetracyclines including tetracycline, doxycycline, and minocycline remained below 3\%, and the resistance rates for macrolides including josamycin, roxithromycin, clarithromycin, and azithromycin were approximately $20 \%$. Acquired tetracycline resistance is associated with the presence of the tet gene [23], while acquired macrolide resistance is related to mutations in the $23 \mathrm{~S}$ rRNA region and variations in ribosomal proteins L4 and L22 [24].

The general increasing trend of antibiotic resistance over the past four years is concerning, and none of the antibiotics showed an obvious decreasing trend. A position statement from the European Sexually Transmitted Infection Guidelines Editorial Board noted that routine detection and treatment of asymptomatic or symptomatic genital Mycoplasma infection in men and women are not recommended because the majority of individuals with asymptomatic infection by these bacteria do not develop disease and excessive detection and treatment may result in the development of antimicrobial resistance [25]. However, a few studies have investigated unresolved issues regarding genital Mycoplasma infection and its independent relationships with sexual risk behaviors, which can result in sexually transmitted diseases (STDs), and/or prostitution [26] and complications such as infertility and pelvic inflammatory disease [27, 28]. Antibiotic treatment can eliminate genital Mycoplasma infection; however, elimination failure may lead to persistent symptoms and signs. Therefore, high-risk populations, such as sex workers and symptomatic patients with genital Mycoplasma infection, should be treated with appropriate antibiotics after informed consent is obtained.

\section{Conclusions}

In conclusion, this study showed increasing trends in the prevalence of infection and levels of antibiotic resistance in U. urealyticum and M. hominis from 2015 to 2018, which clearly confirms the necessity to monitor the standardized administration of antibiotics.

\section{Data Availability}

The datasets used or analyzed during the current study are available from the corresponding author on reasonable request.

\section{Conflicts of Interest}

The authors declare that they have no conflicts of interest.

\section{Acknowledgments}

This study was supported by the Science and Technology Commission Foundation of Chongqing (cstc2018jscxmsybX0107).

\section{References}

[1] J. I. Glass, E. J. Lefkowitz, J. S. Glass, C. R. Heiner, E. Y. Chen, and G. H. Cassell, "The complete sequence of the mucosal pathogen Ureaplasma Urealyticum," Nature, vol. 407, no. 6805, pp. 757-762, 2000.

[2] H. H. Kletzel, R. Rotem, M. Barg et al., "Ureaplasma urealyticum: the role as a pathogen in women's health, a systematic review," Current Infectious Disease Reports, vol. 20, no. 9, p. 33, 2018.

[3] M. A. Francesco, R. Negrini, G. Pinsi, L. Peroni, and N. Manca, "Detection of Ureaplasma biovars and polymerase chain reaction-based subtyping of Ureaplasma parvum in women with or without symptoms of genital infections," European Journal of Clinical Microbiology \& Infectious Diseases, vol. 28, no. 6, pp. 641-646, 2009.

[4] D. G. Tsevat, H. C. Wiesenfeld, C. Parks, and J. F. Peipert, "Sexually transmitted diseases and infertility," American Journal of Obstetrics and Gynecology, vol. 216, no. 1, pp. 1-9, 2017.

[5] K. B. Waites, R. L. Schelonka, L. Xiao, P. L. Grigsby, and M. J. Novy, "Congenital and opportunistic infections: ureaplasma species and Mycoplasma hominis," Seminars in Fetal and Neonatal Medicine, vol. 14, no. 4, pp. 190-199, 2009.

[6] D. Skiljevic, D. Mirkov, and J. Vukicevic, "Prevalence and antibiotic susceptibility of Mycoplasma hominis and Ureaplasma urealyticum in genital samples collected over 6 years at a Serbian university hospital," Indian Journal of Dermatology, Venereology, and Leprology, vol. 82, no. 1, pp. 37-41, 2016.

[7] M. Vargovic, M. Pasini, N. Papic et al., "Antimicrobial susceptibility of Ureaplasma urealyticum and Mycoplasma hominis," Sexually Transmitted Infections, vol. 90, no. 1, p. 69, 2014.

[8] L. Duffy, J. Glass, G. Hall et al., "Fluoroquinolone resistance in Ureaplasma parvum in the United States," Journal of Clinical Microbiology, vol. 44, no. 4, pp. 1590-1591, 2006.

[9] M. Y. Lee, M. H. Kim, W. I. Lee, S. Y. Kang, and Y. L. Jeon, "Prevalence and antibiotic susceptibility of Mycoplasma hominis and Ureaplasma urealyticum in pregnant women," Yonsei Medical Journal, vol. 57, no. 5, pp. 1271-1275, 2016.

[10] C. Leli, A. Mencacci, J. C Bombaci et al., "Prevalence and antimicrobial susceptibility of Ureaplasma urealyticum and Mycoplasma hominis in a population of Italian and immigrant outpatients," Le Infezioni in Medicina, vol. 20, no. 2, pp. 82-87, 2012. 
[11] R. Revello, M. J. Alcaide, D. Abehsera et al., "Prediction of chorioamnionitis in cases of intraamniotic infection by ureaplasma urealyticum in women with very preterm premature rupture of membranes or preterm labour," The Journal of Maternal-Fetal \& Neonatal Medicine, vol. 31, no. 14, pp. 1839-1844, 2018.

[12] Q.-Y. Wang, R.-H. Li, L.-Q. Zheng, and X.-H. Shang, "Prevalence and antimicrobial susceptibility of Ureaplasma urealyticum and Mycoplasma hominis in female outpatients, 2009-2013," Journal of Microbiology, Immunology and Infection, vol. 49, no. 3, pp. 359-362, 2016.

[13] C. C. Ezeanya, N. R. Agbakoba, I. B. Enweani, and C. Oguejiofor, "Predominance of cervicitis agents with minimal testing rate within the student population in Benin city, Nigeria," Journal of Obstetrics and Gynaecology, vol. 39, no. 6, pp. 840-844, 2019.

[14] Y. Zhang, C. Hua, and S. L. Li, "The relationship between the biovars and the antimicrobial resistance of Ureaplasma urealyticum in female patients with urogenital infections," Journal of Clinical Laboratory Analysis, vol. 32, no. 1, Article ID e22211, 2018.

[15] W.-H. Xu, J.-J. Chen, Q. Sun et al., "Chlamydia trachomatis,Ureaplasma urealyticum and Neisseria gonorrhoeaeamong Chinese women with urinary tract infections in Shanghai: a community-based cross-sectional study," Journal of Obstetrics and Gynaecology Research, vol. 44, no. 3, pp. 495-502, 2018.

[16] C. Leli, A. Mencacci, M. A. Latino et al., "Prevalence of cervical colonization by Ureaplasma parvum, Ureaplasma urealyticum, Mycoplasma hominis and Mycoplasma genitalium in childbearing age women by a commercially available multiplex real-time PCR: an Italian observational multicentre study," Journal of Microbiology, Immunology and Infection, vol. 51, no. 2, pp. 220-225, 2018.

[17] Y. Kawai, Y. Nakura, T. Wakimoto et al., "In VitroActivity of five quinolones and analysis of the quinolone resistance-determining regions ofgyrA, gyrB, parC, and parEin ureaplasma parvum and ureaplasma urealyticum clinical isolates from perinatal patients in Japan," Antimicrobial Agents and Chemotherapy, vol. 59, no. 4, pp. 2358-2364, 2015.

[18] X. Xie and J. Zhang, "Trends in the rates of resistance of Ureaplasma urealyticumto antibiotics and identification of the mutation site in the quinolone resistance-determining region in Chinese patients," FEMS Microbiology Letters, vol. 259, no. 2, pp. 181-186, 2006.

[19] M. Mihai, N. Valentin, D. Bogdan, C. M. Carmen, B. Coralia, and S. Demetra, "Antibiotic susceptibility profiles of Mycoplasma hominis and Ureaplasma urealyticum isolated during a population-based study concerning women infertility in northeast Romania," Brazilian Journal of Microbiology, vol. 42, no. 1, pp. 256-260, 2011.

[20] J. B. Choi, S.-J. Lee, M.-K. Lee et al., "Prevalence and antimicrobial susceptibility of Ureaplasma spp. and Mycoplasma hominis in asymptomatic individuals in korea," Microbial Drug Resistance, vol. 24, no. 9, pp. 1391-1396, 2018.

[21] M. L. Beeton, V. J. Chalker, S. Kotecha, and O. B. Spiller, "Comparison of full gyrA, gyrB, parC and parE gene sequences between all Ureaplasma parvum and Ureaplasma urealyticum serovars to separate true fluoroquinolone antibiotic resistance mutations from non-resistance polymorphism," Journal of Antimicrobial Chemotherapy, vol. 64, no. 3, pp. 529-538, 2009.

[22] N. Wang, W. C. Liu, Y. H. Zhou et al., "In vitro activities of nemonoxacin and other antimicrobial agents against human Mycoplasma and Ureaplasma isolates and their defined resistance mechanisms," Frontiers Microbiology, vol. 10, Article ID 01890, 2019.

[23] A. Meygret, C. Le Roy, H. Renaudin, C. Bébéar, and S. Pereyre, "Tetracycline and fluoroquinolone resistance in clinical Ureaplasma spp. and Mycoplasma hominis isolates in France between 2010 and 2015," Journal of Antimicrobial Chemotherapy, vol. 73, no. 10, pp. 2696-2703, 2018.

[24] B. Resch, C. Gutmann, F. Reiterer, J. Luxner, and B. Urlesberger, "Neonatal Ureaplasma urealyticum colonization increases pulmonary and cerebral morbidity despite treatment with macrolide antibiotics," Infection, vol. 44, no. 3 , pp. 323-327, 2016.

[25] P. Horner, G. Donders, M. Cusini, M. Gomberg, J. S. Jensen, and M. Unemo, "Should we be testing for urogenital Mycoplasma hominis, Ureaplasma parvum and Ureaplasma urealyticumin men and women?-a position statement from the European STI Guidelines Editorial Board," Journal of the European Academy of Dermatology and Venereology, vol. 32, no. 11, pp. 1845-1851, 2018.

[26] A. Belkum, C. Schee, W. I. Meijden, H. A. Verbrugh, and H. J. F. Sluiters, "A clinical study on the association of Trichomonas vaginal is and Mycoplasma hominis infections in women attending a sexually transmitted disease (STD) outpatient clinic," FEMS Immunology \& Medical Microbiology, vol. 32, no. 1, pp. 27-32, 2001.

[27] D. Taylor-Robinson, "Mollicutes in vaginal microbiology: mycoplasma hominis, Ureaplasma urealyticum, Ureaplasma parvum and Mycoplasma genitalium," Research in Microbiology, vol. 168, no. 9-10, pp. 875-881, 2017.

[28] M. H. Ahmadi, A. Mirsalehian, and A. Bahador, "Prevalence of urogenital Mycoplasmas in Iran and their effects on fertility potential: a systematic review and Meta-analysis," Iranian Journal of Public Health, vol. 45, no. 4, pp. 409-422, 2016. 